\title{
Deciphering the Pathogenic Nature of Two de novo Sequence Variations in a Patient with Shprintzen-Goldberg Syndrome
}

\author{
Priyanka Srivastava $^{\mathrm{a}}$ Shashank Shende ${ }^{\mathrm{b}}$ Kausik Mandal ${ }^{\mathrm{b}}$ \\ aGenetic Metabolic Unit, Department of Pediatrics, Advanced Pediatric Centre, Post Graduate Institute of Medical \\ Education and Research, Chandigarh, India; 'bepartment of Medical Genetics, Sanjay Gandhi Post Graduate \\ Institute of Medical Sciences, Lucknow, India
}

\section{Keywords}

de novo $\cdot$ Novel $\cdot$ Co-occurrence $\cdot$ Shprintzen-Goldberg

syndrome $\cdot$ Sanger sequencing rise to SGS. This case illustrates the issues regarding the importance and difficulties associated with the determination of the causative variations in a single-gene disorder.

(c) 2021 S. Karger AG, Basel

\section{Introduction}

Shprintzen-Goldberg syndrome (SGS; OMIM \# 182212) includes virtually all of the craniofacial, skeletal, skin, and cardiovascular manifestations of Marfan syndrome (MFS) and Loeys-Dietz syndrome (LDS), additionally with intellectual disability and severe skeletal muscle hypotonia [Doyle et al., 2012]. On the basis of previous published cases, SGS is an autosomal dominant (AD) disorder. In a rare intance, recurrence has been reported in siblings [Adès et al., 1995].

In last few decades, it has become more clear that dysregulated transforming growth factor beta (TGF $\beta$ ) signalling plays an important role in the pathogenesis of MFS, LDS, and other related disorders involving thoracic aortic aneurysms. Based on the overlapping features of MFS and LDS and also the evident key role of TGF $\beta$ signalling in the pathogenesis of MFS and LDS, it was hypothesized karger@karger.com

(C) 2021 S. Karger AG, Basel

www.karger.com/msy

Karger ${ }^{\prime \prime}=$
Correspondence to:

Kausik Mandal, mandal.kausik@gmail.com 
that altered TGF $\beta$ signalling can play some role in the SGS pathogenesis. Mutations in the SKI gene (encoding the oncoprotein SKI, a repressor of TGF $\beta$ activity) were shown to be causative of SGS. SKI is an avian sarcoma viral oncogene homolog that is located in chromosome 1 and consists of 7 exons. Here, we present a 12-year-old female child with features suggestive of SGS. Based on the clinical phenotype, we sequenced SKI, and the findings were unusual. There was co-occurrence of 2 heterozygous missense sequence variations, c.346G $>$ A (p.Gly116Arg) and c.687G>C (p.Lys229Asn), in exon 1 (hotspot) of the SKI gene.

\section{Case Report}

A 12-year-old girl presented to the outpatient department with major dysmorphic features noticed since 5 years of age. She had an extremely thin body habitus along with abnormally long and apparently disfigured bones. She was born full term by normal vaginal delivery to nonconsanguineous parents after an uneventful pregnancy. Her birth weight was $2.5 \mathrm{~kg}$. Body size and habitus were apparently normal until 5 years of age, but global developmental delay was noticed. She started sitting without support at approximately 15 months of age and walking without support at 2 and half years of age. She started talking using simple sentences at around 5 years of age. She is the first born of 4 children. Her family history was insignificant with no other affected siblings or family members. Her past medical and surgical history also was insignificant. On examination, her weight was 25 $\mathrm{kg}(-3 \mathrm{SD})$, height was $152 \mathrm{~cm}$ (50th centile) and head circumference was $51 \mathrm{~cm}$ (5th centile). She had a characteristic facies as a consequence of craniosynostosis of the sagittal and coronal sutures, including hypertelorism, downslanting palpebral fissures, proptosis, low-set ears, malar hypoplasia, high-arched palate, and micrognathia (Fig. 1a). Pectus carinatum, arachnodactyly, scoliosis, joint hypermobility with bilateral elbow dislocations, arachnodactyly and flat feet were found. X-ray of the skull showed evidence of craniosynostosis (Fig. 1b). Further Xrays showed long narrow thorax with tubular heart, angulation of sternum, kyposcoliosis of spine, pelvis-tilt associated with scoliosis, bilateral elbow dislocation (dislocation of ulna), and arachnodactyly (Fig. 1 c, d, e). There was no protrusio acetabuli, which is a feature of MFS. Echocardiography showed mild mitral valve prolapse, trivial tricuspid regurgitation, but no aortic regurgitation or mitral regurgitation. Ophthalmological examination showed mild dilated pupils with sluggish light reactions. There was no lens subluxation. Ocular movement was full. She required medical follow-up for the last 4 years. Her IQ was 65, and she had difficulties articulating properly when speaking. At 16 years of age, at present, she is studying in 10th standard.

\section{Materials and Methods}

Blood samples from both the unaffected parents and proband were collected in EDTA vacutainer. Genomic DNA from the blood samples was extracted using QIAamp DNA Blood extraction kit (Germany).

Sanger sequencing of coding regions and conserved splice sites of the SKI gene was done. PCR was performed using standard conditions on a thermal cycler (Applied Biosystems, Foster City, CA, USA). PCR products were bidirectionally sequenced using the BigDye Terminator Cycle Sequencing kit (Applied Biosystems) and separated on an ABI 3130 Genetic Analyzer in accordance with the manufacturer's instructions (Applied Biosystems), followed by bioinformatic analysis using various softwares. Protein modelling was done to decipher the pathogenicity of the variants using in silico tools.

\section{Results}

\section{Mutation Analysis}

Considering the AD mode of inheritance, heterozygous variation was expected. Interestingly, we identified 2 different missense heterozygous variants (c.346G $>$ A; p.Gly116Arg) and (c.687G>C; p.Lys229Asn) in exon 1 of SKI (hotspot) in the same propositus (shown in Fig. 2a, b). At the time of initial analysis, both were predicted as novel variants and were found to be disease causing and damaging by MutationTaster(http://www.mutationtaster.org/) and SIFT (http://provean.jcvi.org/index. php). The p.Gly116Arg was not reported in dbSNP, 1000 Genomes databases (http://www.1000genomes.org), exome variant server (http://evs.gs.washington.edu/ EVS/), or ExAC database (http://exac.broadinstitute. org/). Recently, the p.Lys229Asn variant was found to be reported in Gnomad (https://gnomad.broadinstitute. org/) with a frequency of $<0.01(0.000004101)$. We concluded that this variant could be likely benign. Further, both variants were tested in the parents, but both sequence variations were found to be de novo. QF-PCR was done to check parentage; both father and mother were the biological parents. To rule out polymorphism in our population, we have done the sequencing for these variations in 50 control samples. None of the varaints were detected in control samples. p.Gly116Arg was found to be conserved among different species, whereas p.Lys229Asn 


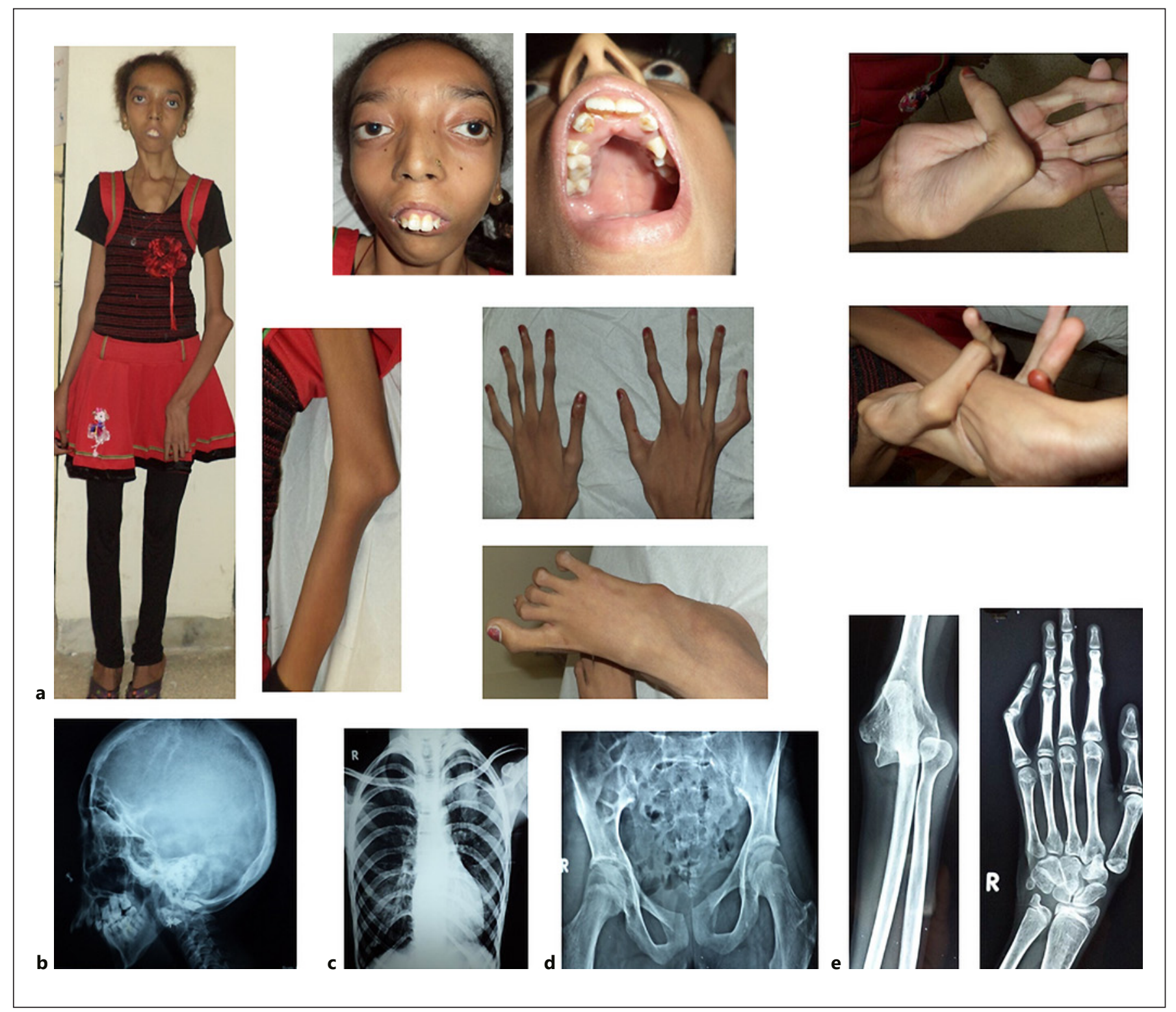

Fig. 1. a Clinical photographs of the patient showing characteristic facies with hypertelorism, downslanting palpebral fissures, low-set ears, malar hypoplasia, proptosis, high-arched palate, micrognathia, pectus carinatum, arachnodactyly, scoliosis, joint hypermobility, elbow joint dislocations, flat foot. b Lateral X-ray of the skull showing craniosynostosis of the coronal sutures causing restric- tion of anteroposterior growth of the upper part of the skull and in turn leading to protrusion of the upper jaw and upper incisor teeth. c X-ray shows long, narrow thorax with tubular heart and kyposcoliosis of spine. d Pelvis tilt associated with scoliosis of the spine, no protrusio acetabuli. e Arachnodactyly. was not. According to ACMG criteria, both variants are classified as "variants of unknown significance." For Gly116Arg, the scores are 2 moderate and 1 supporting (PM2, PM5, and PP3), and for Lys229Asn it is 1 moderate and 1 supporting (PM2 and PP3) only. In view of these findings, we assumed that p.Gly116Arg is the likely causative variant in our patient.

Two SKI Variants in One Patient with Shprintzen-Goldberg Syndrome

\section{Protein Modeling}

As both the variations were predicted to be novel and pathogenic by in silico tools, protein modelling was done to decipher the pathogenicity of the 2 mutations. Secondary structure analysis of SKI was performed on Predict Protein server accessed as https://www.predictprotein. org. Both of the variants are located far apart, so 2 differ-

Mol Syndromol 2021;12:141-147 DOI: $10.1159 / 000514125$ 


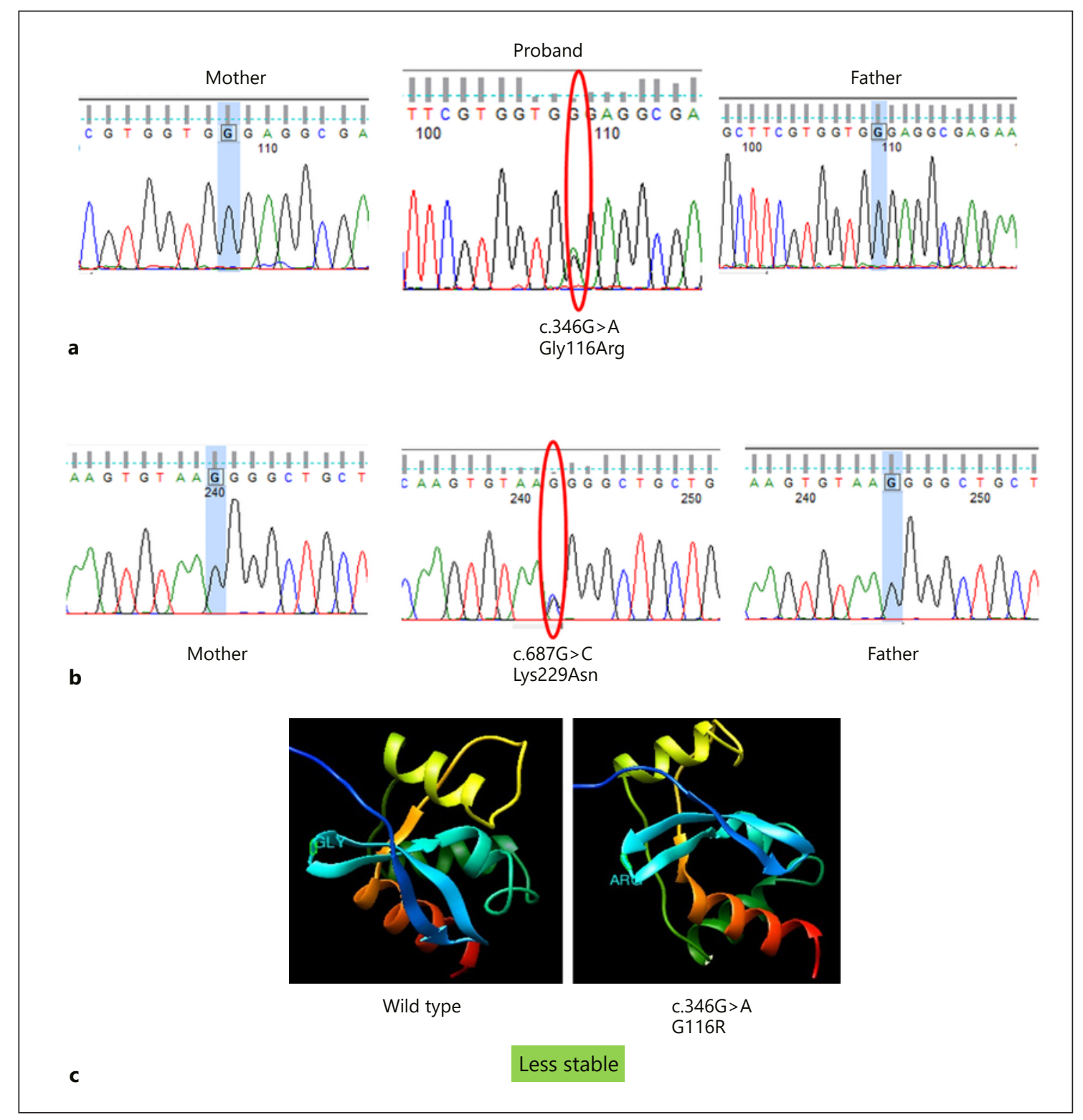

Fig. 2. a Chromatogram of the proband showing variant c.346G>A, mother and father carrying the wild-type allele. b Proband showing the c.687G $>C$ variant and parents carrying the wild-type allele. c Protein modelling of the SKI gene showing wild type and mutant c.346G>A (p.Gly116Arg). Protein stability test revealed that the mutant is less stable than the wild type.

ent templates were selected for both. The closest template, identified and retrieved from the protein data bank (PDB), for both wild and mutant SKI (p.Gly116Arg) was that of the SKI oncogene (PDB id: 1sbx.1.A) of Homo sapiens [Mitsiki et al., 2009]. For another variant, p.Lys229Asn, wild and mutant template was identified as $1 \mathrm{mr1.2B}$. Homology models of deduced SKI protein was constructed on SWISSMODEL workspace (shown in Fig. 2c) [Arnold et al., 2006] and finally visualized using
University of California, San Francisco (UCSF) Chimera [Pettersen et al. 2004]. Further, the quality of the protein model was evaluated by QMEAN4 and PROCHECK [Laskowskiet al. 1993].

Ramachandran Plot statistics of generated homology model of the wild-type SKI (1sbx.1.A) showed that $92.6 \%$ residues were in most favored regions, $7.4 \%$ residues were in additionally allowed regions, while no residues were in generously and disallowed allowed regions. QMEAN 
Table 1. Predictions of different softwares for predicting protein stability of the mutants

\begin{tabular}{llllllll}
\hline AA change & Inheritance & I-mutant 2.0 & $\begin{array}{l}\mathrm{mCSM} \\
\mathrm{kcal} / \mathrm{mol}\end{array}$ & $\begin{array}{l}\text { SDM } \\
\mathrm{kcal} / \mathrm{mol}\end{array}$ & $\begin{array}{l}\text { DUET } \\
\mathrm{kcal} / \mathrm{mol}\end{array}$ & QMEAN4 & Prediction \\
\hline G116R & $\mathrm{AD}$ & -0.40 & -0.345 & -2.17 & -0.545 & -0.01 & Destabilizing \\
K229N & $\mathrm{AD}$ & -0.43 & - & - & - & - & - \\
\hline
\end{tabular}

Two-stage prediction classification: destabilizing ( $<0 \mathrm{kcal} / \mathrm{mol})$, stabilizing $(>0 \mathrm{kcal} / \mathrm{mol})$; AD, autosomal dominant

score of wild type SKI (1sbx.1.A) was 0.12 and Overall (PROCHECK) score was 0.46. The main-chain G-factor for wild-type $S K I$ was found to be 0.59 , which is greater than the acceptable value -0.50 . Analysis of mutant $\mathrm{p}$. Gly116Arg showed that $92.4 \%$ residues were in most favored regions with QMEAN4 of -0.01 and overall PROCHECK score of 0.28 . Main-chain $\mathrm{G}$-factor value was 0.43 , which is lower than that of the wild type. Unstable models are expected to have strongly negative $Z$ scores for QMEAN. It shows that mutant p.Gly116Arg (QMEAN score -0.01) is less stable than the wild-type SKI (QMEAN score 0.12). It can affect the overall confirmation of the SKI protein.

For the second variation, p.Lys229Asn, a good quality model could not be made because of the low sequence identity and coverage of the template. The Ramachandran analysis showed the model to be too unstable to interpret.

\section{Protein Stability Analysis}

For computational stability analysis, we relied on the $3 \mathrm{D}$ protein structure of SKI, which was implemented into the structure-based prediction tools mutation cutoff scanning matrix (mCSM), site-directed mutator (SDM), DUET, and the 3-state predictor I-mutant 2.0. Accordingly, the analyzed pathologic mutation (Gly116Arg) was classified as destabilizing, noticeable by a large decline in Gibbs free energy as indicated by a negative DDG value (Table 1).

\section{Cis-Trans Testing}

We assumed that the mutations are in the same allele (in cis). As per SNAPGENE (https://www.snapgene.com/ snapgene-viewer/), at the position of the second mutation (c.687G >C; p.Lys229Asn) when $\mathrm{G}$ is replaced by C (GTAAG to GTAAC), a restriction site is formed at the site of the sequence change $(\mathrm{G}>\mathrm{C})$ for the restriction enzyme MaeIII.

As both the sequence variants are nearby, we designed a set of primers (Details: F: TGCTGCACCTGCCCGC-
CATCCA and R: CTTCGGAGACCAGAGCCTGTA) to amplify both variations in one PCR reaction. The product length was $835 \mathrm{bp}$. After PCR amplification, digestion was done with the restriction enzyme MaeIII. After digestion, 1 allele with 835-bp band was divided into 2 products with 465-bp and 370-bp bands (online suppl. Fig. 1; for all suppl. material, see www.karger.com/ doi/10.1159/514125). The undigested and digested product of 835 was sequenced bidirectionally to look for the 2 sites of sequence change (online suppl. Fig. 2, 3). The 465bp band was sequenced by the forward primer to look for the proximal mutant site (online suppl. Fig. 4).

As per our assumption, the undigested PCR product showed both heterozygous peaks (online suppl. Fig. 2), and after digestion at the distal mutation site, the 835-bp product showed both wild peaks (online suppl. Fig. 3), and the digested 465-bp band showed the mutant peak (online suppl. Fig. 4) after sequencing.

\section{Discussion and Conclusion}

The clinical picture of the proband having SKI mutations is compatible with previously reported patients. Typical craniofacial features include craniosynostosis with proptosis, dolichocephaly with retrognathia, hypertelorism, low-set ears, and the absence of ectopia lentis. Skeletal findings include arachnodactyly, scoliosis, and a combination of joint hyperlaxity and contractures with camptodactyly. The girl showed moderate developmental delay and intellectual disability.

All SKI mutations occurred within exon 1, and comprise approximately $90 \%$ of clinically diagnosed SGS. Our patient carries a mutation in exon 1 , but the unusual part is co-occurrence of 2 de novo novel missense sequence variants: c.346G >A (p.Gly116Arg) and c.687G >C (p.Lys229Asn). A mutation at position 116 (p.Gly116Glu) has already been reported in the literature as pathogenic [Doyle et al., 2012], but our patient has a change of glycine 
Table 2. Prediction scores and population frequencies of mutations using in silico softwares

\begin{tabular}{llllllll}
\hline Gene & Transcript ID & Mutation & $\begin{array}{l}\text { Amino } \\
\text { acid } \\
\text { change }\end{array}$ & Mutationtaster & SIFT & Polyphen & ExAC frequency \\
\hline SKI & NM_003036.3 & c.346G>A & G116R & Disease causing, score: 125 & Damaging; score: 0 & Probably damaging; score: 1.00 & Not reported \\
\cline { 2 - 8 } & & c.687G>C & K229N & Disease causing, score: 94 & Damaging; score: 0.02 & Possibly damaging; score: 0.992 & 0.000009370 \\
(rare; VOUS) & & & & & & &
\end{tabular}

to arginine at 116 position. This substitution is present at a highly conserved residue in an exposed beta hairpin loop in the DHD domain. Mostly SKI mutations have been reported between residues 21-117, and recurrent mutations affecting certain amino acid residues, such as 35 and 116, have become evident [Au et al., 2014]. This mutational hot spot indicates that this region is critical for protein interactions. The residues between $17-45$ and $125-131$ present in the receptor-mediated SMAD- (R-SMAD) binding domain [Wu et al., 2002], suggest that altered SKI function in SGS may be a reason for impaired interaction with RSMADs. This idea is supported by altered TGF $\beta$ signals in patient-derived fibroblasts with confirmed exon 1 SKI missense mutations, with increased phosphorylation of RSMADs and ERK and increased transcription of TGF $\beta$ dependent genes [Doyle et al., 2012].

The second sequence variant found in our patient (p.Lys229Asn) is present in the SMAD4-binding domain. The crystal structure of the SMAD4-binding domain revealed specific recognition of the SMAD4 L3 loop region by a highly conserved interaction loop (I loop) from SKI [Wu et al., 2002]. The SKI-binding surface on SMAD4 was found to significantly overlap with that required for binding of the R-SMADs. Indeed, SKI disrupted the formation of a functional complex between the co-mediator SMADs and R-SMADs, explaining how it could lead to repression of TGF $\beta$, active in bone morphogenetic protein responses.

So far 17, different SKI mutations have been identified in 33 unrelated patients ( 1 patient was identical in ref Doyle et al. [2012] and Carmignac et al. [2012]). Schepers et al. [2015] analyzed the SKI gene in 19 patients and identified 8 recurrent and 3 novel mutations in 11 patients in the hotspot region of SKI. In contrast to the previous reports, we identified 2 de novo mutations in a single patient. Furthermore, to collect the more pathogenicity evidences, we performed homology modelling for both variants. Based on the homology modelling mutant, p. Gly116Arg showed to affect the protein stability and confirmation. All the in silico tools predicted both variants as likely pathogenic, but p.Gly116Arg was found to be more pathogenic as compared to p.Lys229Asn by different scoring of in silico tools as well by protein modelling (Table 2). Functional studies are warranted to prove the pathogenic nature of the variants and to analyze the contribution of each variation in disease pathogenesis, which is the limitation of our study. This case illustrates the issues regarding the importance and difficulties associated with the determination of the causative variations in a single-gene disorder. We however assume that $\mathrm{p}$. Gly116Arg can explain the phenotype and that p.Lys229Asn is a rare simultaneous spontaneous event and is likely to be a polymorphism in cis.

Spontaneous mutations are predicted to be higher in eukaryotes [Drake et al., 1998], and there are various ways to determine spontaneous mutations [Foster, 2006]. As could be predicted, a single spontaneous mutation in a single gene such as $S K I$ is likely to be approximately $10^{-6}$; for 2 mutations to occur, the chance is about $10^{-12}$. Our case is a unique example of the very rare event of 2 spontaneous mutations in a single gene.

\section{Acknowledgement}

We sincerely thank the cooperation of patient families and acknowledge the Department of Science and Technology (SR/WOSA/LS-48/2016).

\section{Statement of Ethics}

This study is approved by the Ethical Committee of the institute. Written Informed consent was obtained from the patient and her parents for the molecular genetic analysis and publication of accompanying images.

\section{Conflict of Interest Statement}

There are no conflicts of interest to declare. 


\section{Funding Source}

The study was funded by the Department of Science and Technology (SR/WOS-A/LS-48/2016).

\section{Author Contributions}

K. Mandal: Substantial contributions to the conception and design of the work, clinical assessment of the patient, drafting the work, and revising it critically for important intellectual content. P. Srivastava: Performed experiments, analyzed and interpreted data for the work, drafted the manuscript. S. Shende: Performed laboratory work and experiments.

\section{References}

Adès LC, Morris LL, Power RG, Wilson M, Haan EA, Bateman JF, et al. Distinct skeletal abnormalities in four girls with Shprintzen-Goldberg syndrome. Am J Med Genet. 171995; 57(4):565-72.

Arnold K, Bordoli L, Kopp J, Schwede T. The SWISS-MODEL workspace: a web-based environment for protein structure homology modelling. Bioinformatics. 2006;22(2):195201.

Au PY, Racher HE, Graham JM Jr, Kramer N, Lowry RB, Parboosingh JS, et al. De novo exon 1 missense mutations of SKI and Shprintzen-Goldberg syndrome: two new cases and a clinical review. Am J Med Genet A. 2014;164A(3):676-84.
Carmignac V, Thevenon J, Adès L, Callewaert B, Julia S, Thauvin-Robinet $C$, et al. In-frame mutations in exon 1 of SKI cause dominant Shprintzen-Goldberg syndrome. Am J Hum Genet. 2012;91(5):950-7.

Doyle AJ, Doyle JJ, Bessling SL, Maragh S, Lindsay ME, Schepers D, et al. Mutations in the TGF- $\beta$ repressor SKI cause Shprintzen-Goldberg syndrome with aortic aneurysm. Nat Genet. 2012;44(11):1249-54.

Drake JW, Charlesworth B, Charlesworth D, Crow JF. Rates of spontaneous mutation. Genetics. 1998;148(4):1667-86.

Foster PL. Methods for Determining Spontaneous Mutation Rates. Methods Enzymol. 2006; 409:195-213.

Laskowski RA, MacArthur MW, Moss DS, Thornton JM. PROCHECK: a program to check the stereochemical quality of protein structures. J Appl Cryst. 1993;26(2):283-91.
Mitsiki E, Papageorgiou AC, Iyer S, Thiyagarajan $\mathrm{N}$, Prior SH, Sleep D, et al. Structures of native human thymidine phosphorylase and in complex with 5-iodouracil. Biochem Biophys Res Commun. 2009;386(4):666-70.

Pettersen EF, Goddard TD, Huang CC, Couch GS, Greenblatt DM, Meng EC, et al. UCSF Chimera--a visualization system for exploratory research and analysis. J Comput Chem. 2004;25(13):1605-12.

Schepers D, Doyle AJ, Oswald G, Sparks E, Myers L, Willems PJ, et al. The SMAD-binding domain of SKI: a hotspot for de novo mutations causing Shprintzen-Goldberg syndrome. Eur J Hum Genet. 2015;23(2):224-8.

Wu JW, Krawitz AR, Chai J, Li W, Zhang F, Luo $\mathrm{K}$, et al. Structural mechanism of Smad4 recognition by the nuclear oncoprotein Ski: insights on Ski-mediated repression of TGF-beta signaling. Cell. 2002;111(3):357-67. 